\title{
INSTABILITY OF SPHERES WITH DEFORMED RIEMANNIAN METRICS
}

\author{
By SHUKICHI TANNO
}

\section{$\S 1$. Introduction.}

Let $(M, g)$ be a compact Riemannian manifold. Then $(M, g)$ is said to be unstable, if the identity map $i d_{M}$ of $(M, g)$ is unstable as a harmonic map; that is, the Jacobi operator $J$ coming from the second variation of the energy functional at $i d_{M}$ has negative eigenvalues. The standard sphere $\left(S^{m}, g_{0}\right)$ of constant curvature 1 is unstable for $m \geqq 3$. Furthermore, unstable, simply connected compact (irreducible) symmetric spaces were determined (Smith [10], Nagano [5], Ohnita [7], Urakawa [17]).

In this note, as a class of homogeneous Riemannian manifolds which are not symmetric nor Einstein, we study $\left(S^{m}, g(t)\right)$ with $m=2 n+1$. Here $g(t)$ is defined as follows: For $m=2 n+1$, we have the Hopf fibration $\pi:\left(S^{m}, g_{0}\right) \rightarrow$ $\left(C P^{n}, h_{0}\right)$, where $\left(C P^{n}, h_{0}\right)$ denotes the complex projective $n$-space with the Fubini-Study metric of constant holomorphic sectional curvature 4. Let $\xi$ be a vector field on $S^{m}$ which is tangent to the fibers and of unit length. $\xi$ is a Killing vector field with respect to $g_{0}$ and the 1 -form $\eta$ dual to $\xi$ with respect to $g_{0}$ defines a canonical contact structure on $S^{m}$. Then a 1-parameter family of Riemannian metrics $g(t)$ on $S^{m}$ is defined by

$$
g(t)=t^{-1} g_{0}+t^{-1}\left(t^{m}-1\right) \eta \otimes \eta
$$

where $0<t<\infty$ (Urakawa [16], Tanno [13]). With respect to these Riemannian metrics, the volume element is unchanged.

We prove the following.

THEOREM. For $m=2 n+1 \geqq 3$ and $t \in\left(t_{0}(m), \infty\right),\left(S^{m}, g(t)\right)$ is unstable, where $t_{0}(m)=\left[\left[\left(m^{2}-4\right)^{1 / 2}-1\right] /\left(m^{2}-5\right)\right]^{1 / m}$ and $t_{0}(3)=0.67 \cdots<t_{0}(m)<1$. For each eigenfunction $f$ corresponding to the first eigenvalue $m$ of the Laplacian of $\left(S^{m}, g_{0}\right)$,

$$
f \xi+\left[\left\{m-2 t^{m}+\left[\left(2 t^{m}-1\right)^{2}+m^{2}-1\right]^{1 / 2}\right\} / 2(m-1)\right] \nabla_{\mathbf{g r a d} f} \xi
$$

is an eigen vector corresponding to the negative eigenvalue $\mu(t)(c f .(3.4))$ of the Jacobi operator $J(t)$.

Received March 12, 1987 


\section{§ 2. Preliminaries.}

Let $\eta$ be a canonical contact structure on $S^{m}, m=2 n+1 \geqq 3$, and $\xi$ be its dual with respect to $g=g_{0}$. In this section, we write $g$ instead of $g_{0}$ for simplicity. Then $(\phi, \xi, \eta, g)$ is a Sasakian structure, where $\phi=-\nabla \xi$. The structure tensors satisfy the following relations:

$$
\begin{aligned}
& \phi \xi=0, \quad \eta \cdot \phi=0, \quad \eta(\xi)=1, \\
& \phi \phi X=-X+\eta(X) \xi, \\
& g(X, Y)=g(\phi X, \phi Y)+\eta(X) \eta(Y), \\
& \left(\nabla_{X} \phi\right)(Y)=g(X, Y) \xi-\eta(Y) X,
\end{aligned}
$$

where $X$ and $Y$ are vector fields on $S^{m}$.

If $m=4 r+3$, then we have Killing vector fields $\xi_{(\alpha)}, \alpha=1,2,3$, which are orthonormal and satisfy

$$
\begin{aligned}
& {\left[\xi_{(\alpha)}, \xi_{(\beta)}\right]=2 \xi_{(\gamma)},} \\
& \phi_{(\alpha)} \xi_{(\beta)}=-\phi_{(\beta)} \xi_{(\alpha)}=\xi_{(\gamma)}, \\
& \phi_{(\alpha)} \phi_{(\beta)}-\xi_{(\alpha)} \otimes \eta_{(\beta)}=-\phi_{(\beta)} \phi_{(\alpha)}+\xi_{(\beta)} \otimes \eta_{(\alpha)}=\phi_{(\gamma)},
\end{aligned}
$$

where $(\alpha, \beta, \gamma)$ is a cyclic permutation of $(1,2,3)$, and $\phi_{(\alpha)}$ and $\eta_{(\alpha)}$ are defined analogously.

Let $\lambda_{k}$ be the $k$-th eigenvalue of the Laplacian $\Delta$ acting on functions on $\left(S^{m}, g\right)$ with multiplicity $\nu(k)$. Then

$$
\operatorname{Spec}\left(S^{m}, g\right)=\left\{\lambda_{k}=k(m+k-1) ; k=0,1,2, \cdots\right\}
$$

$\nu(0)=1, \nu(1)=m+1$ and $\nu(k)={ }_{m+k} C_{k}-{ }_{m+k-2} C_{k-2}$ for $k \geqq 2$. Let $V_{k}$ denote the eigenspace corresponding to the eigenvalue $\lambda_{k}$. Then we have the orthogonal decomposition of $V_{k}$;

$$
V_{k}=V_{k, k}+V_{k, k-2}+\cdots+V_{k, k-2[k / 2]},
$$

where $[k / 2]$ is the integral part of $k / 2$, and for $\varphi \in V_{k, \theta}$

$$
L_{\xi} L_{\xi} \varphi+(k-2 p)^{2} \varphi=0
$$

holds for $\theta=k-2 p, 0 \leqq p \leqq[k / 2]$ (Tanno [13], p. 182). Here $L_{\xi}$ denotes the Lie derivation by $\xi$. Let $\varphi \in V_{k, 0}$. Then $L_{\xi} L_{\xi} \varphi=0$ implies $L_{\xi} \varphi=0$ and $\varphi$ is constant along each fiber of the Hopf fibration.

If $m=3$, then $V_{2,0}$ is 3-dimensional and $V_{1}$ is 4-dimensional. By $\left\{f_{(l)}\right\}$ we denote a base of $V_{1}$ or $V_{2,0}$ (cf. [15], p. 122).

Proposition 2.1. The vector space of all Killing vector fields on $\left(S^{3}, g\right)$ is 
spanned by vector fields dual to

$$
\begin{aligned}
& \eta_{(1)}=\eta, \quad \eta_{(2)}, \quad \eta_{(3)}, \\
& 2 f_{(l)} \eta+d f_{(l)} \cdot \phi \quad f_{(l)} \in V_{2,0}, l=1,2,3 .
\end{aligned}
$$

These 1-forms are coclosed eigen 1-forms corresponding to the eigenvalue 4 of the Laplacian.

The vector space of all conformal Killing vector fields on $\left(S^{3}, g\right)$ is spanned by vector fields dual to (2.1), (2.2) and

$$
f_{(l)} \eta+d f_{(l)} \cdot \phi \quad f_{(l)} \in V_{1}, l=1,2,3,4 .
$$

These 1-forms in (2.3) are closed eigen 1-forms corresponding to the eigenvalue 3 of the Laplacian.

Proof. As for eigen 1-forms, see Lemma 2.5 and Proposition 3.1 in [15]. Here, we identified $\nabla_{\xi} d f$ with $d f \cdot \phi$ for $f \in V_{k, 0}$. A direct method to see that 1 -forms in (2.2) define Killing vector fields is to use $\phi=-\nabla \xi$ and the differential equation

$$
\nabla_{k} \nabla_{j} \nabla_{\imath} f+2 \nabla_{k} f g_{\imath j}+\nabla_{\jmath} f g_{i k}+\nabla_{\imath} f g_{j k}=0
$$

satisfied by $f \in V_{2}$ (cf. Obata [6], Tanno [12]). To verify that 1 -forms in (2.3) define conformal Killing vector fields, we use the fact that each $f$ in $V_{1}$ satisfies $\nabla_{\imath} \nabla, f=-f g_{\imath \jmath}$.

q.e.d.

Let $\mathscr{X} M$ be the set of all smooth vector fields and $\Lambda^{1} M$ the set of all smooth 1 -forms on a smooth manifold $M$. By $Q$ we denote the Ricci operator;

$$
\begin{array}{ll}
Q: \mathscr{X} M \rightarrow \mathscr{X} M & \left(X=\left(X^{j}\right) \rightarrow Q X=\left(R_{\jmath}^{\imath} X^{j}\right)\right), \\
Q: \Lambda^{1} M \rightarrow \Lambda^{1} M & \left(w=\left(w_{k}\right) \rightarrow Q w=\left(R_{\jmath}^{k} w_{k}\right)\right),
\end{array}
$$

where $\left(R_{j_{k}}\right)$ denotes the Ricci tensor of a Riemannian manifold $(M, g)$.

Let $J: \mathscr{X} M \rightarrow \mathscr{X} M$ be the Jacobi operator of the identity map as a harmonic map of $(M, g)$ onto $(M, g)$ (Smith [10]). By the natural correspondence between $\mathscr{X} M$ and $\Lambda^{1} M$, in the following we use $J=-\Delta-2 Q: \Lambda^{1} M \rightarrow \Lambda^{1} M$.

$Q=2 I$ holds on $\left(S^{3}, g\right)$, where $I$ denotes the identity. If $w$ is one of 1 -forms in (2.1) and (2.2), then $J w=0$ holds. If $w$ is one of 1 -forms in (2.3), then $J w=$ $-w$ holds. The index Ind $(i d)$ is equal to 4 and (2.3) gives a base for the space of eigen 1-forms corresponding to the negative eigenvalues of $J$. The nullity Null (id) is equal to 6 and (2.1) and (2.2) give a base for the nullity space of $J$. The decomposition in Proposition 2.1 is naturally related to the changing eigen 1-forms of $J$ corresponding to the deformation (1.1) of the Riemannian metrics on $S^{3}$. This situation is explained in Theorem 3.8 in the next section.

The following (i) $\sim(v)$ are proved in $[15]$; 
(i) If $\Delta f+\lambda f=0$ holds on $\left(S^{m}, g\right)$, then

$$
\begin{aligned}
& \Delta(f \eta)=-(\lambda+2 m-2) f \eta+2 d f \cdot \phi, \\
& \Delta(d f \cdot \phi)=2 \lambda f \eta-(\lambda+2) d f \cdot \phi+2 \nabla_{\xi} d f .
\end{aligned}
$$

(ii) If $w$ is $f \eta$ or $d f \cdot \phi$, where $f \in V_{1}$ for $\left(S^{m}, g\right)$, then $L_{\xi} L_{\xi} w=-w$ holds.

(iii) Let $f \in V_{2,0}$ for $\left(S^{m}, g\right)$. Then,

$$
L_{\xi} L_{\xi}(f \eta)=L_{\xi} L_{\xi}(d f \cdot \phi)=0 .
$$

(iv) For a function $f$ on $\left(S^{m}, g\right)$

$$
\begin{aligned}
& \phi^{r s} \nabla_{r}\left(f \eta_{s}\right)=(m-1) f, \\
& \phi^{r s} \nabla_{r}\left(\phi_{s}^{h} \nabla_{h} f\right)=\Delta f-L_{\xi} L_{\xi} f .
\end{aligned}
$$

(v) On $\left(S^{m}, g(t)\right)$ with (1.1) the inverse $\left(g(t)^{r s}\right)$ of $\left(g(t)_{s j}\right)$, the Christoffel's symbols $\Gamma(t)_{j k}^{\imath}$, the Ricci curvature tensor $\left(R_{j k}^{(t)}\right)$ and the Laplacian $\Delta^{(t)}$ are given by

$$
\begin{aligned}
& g(t)^{r s}=t g^{r s}-t\left(1-t^{-m}\right) \xi^{r} \xi^{s}, \\
& \Gamma(t)_{j k}^{\imath}-\Gamma_{j k}^{\imath}=\left(1-t^{m}\right)\left(\phi_{j}^{2} \eta_{k}+\phi_{k}^{\imath} \eta_{j}\right), \\
& R_{j k}^{(t)}=R_{j k}-2\left(t^{m}-1\right) g_{j k}+\left(t^{m}-1\right)\left(m+1+(m-1) t^{m}\right) \eta_{j} \eta_{k}, \\
& \Delta^{(t)} w=t \Delta w-t\left(1-t^{-m}\right) L_{\xi} L_{\xi} w-2 t\left(t^{m}-1\right)\left(\phi^{r s} \nabla_{r} w_{s}\right) \eta, \\
& \Delta^{(t)} \eta=-2(m-1) t^{m+1} \eta, \\
& \Delta^{(t)} \eta_{(\alpha)}=-\left[2(m-3) t+4 t^{1-m}\right] \eta_{(\alpha)} \quad \alpha=2,3,
\end{aligned}
$$

where $w \in \Lambda^{1} S^{m}$.

\section{$\S 3$. The Jacobi operator $J(t)$.}

LEMMA 3.1. The Ricci operator $Q^{(t)}$ on $\left(S^{m}, g(t)\right)$ satısfies the following ;

$$
\begin{aligned}
& Q^{(t)} \eta=(m-1) t^{m+1} \eta, \\
& Q^{(t)} w=t\left(m+1-2 t^{m}\right) w,
\end{aligned}
$$

for $w \in \Lambda^{1} S^{m}$ such that $w(\xi)=0$.

Proof. By (2.4) and (2.6) we obtain

$$
R_{i}^{(t) r}=t\left(m+1-2 t^{m}\right) \delta_{i}^{r}+(m+1) t\left(t^{m}-1\right) \xi^{r} \eta_{\imath},
$$

from which proof is completed. 
LEMMA 3.2. The Jacobi operator $J(t)$ on $\left(S^{m}, g(t)\right)$ is given by

$$
\begin{gathered}
J(t) w=-t \Delta w+t\left(1-t^{-m}\right) L_{\xi} L_{\xi} w+2 t\left(t^{m}-1\right)\left(\phi^{r s} \nabla_{r} w_{s}\right) \eta \\
-2 t\left(m+1-2 t^{m}\right) w-2(m+1) t\left(t^{m}-1\right) w(\xi) \eta
\end{gathered}
$$

for $w \in \Lambda^{1} S^{m}$.

Proof. (3.2) follows from (2.7), (3.1) and the definition of $J(t)$. q.e.d.

Lemma 3.3. Let $f \in V_{1}$ for $\left(S^{m}, g\right)$ and put

$$
w(t)=f \eta+a(t) d f \cdot \phi,
$$

where

$$
a(t)=\left\{m-2 t^{m}+\left[\left(2 t^{m}-1\right)^{2}+m^{2}-1\right]^{1 / 2}\right\} / 2(m-1) t^{m} .
$$

Then, $J(t) w(t)=\mu(t) w(t)$ holds on $\left(S^{m}, g(t)\right)$, where

$$
\mu(t)=2 t^{m+1}+t^{1-m}-t-t\left[\left(2 t^{m}-1\right)^{2}+m^{2}-1\right]^{1 / 2} .
$$

Proof. $J(t) w(t)=\mu(t) w(t)$ is verified by direct calculation, using (3.2), $\nabla_{j} \nabla_{\imath} f$ $=-f g_{\imath \jmath}$, and relations (i), (ii) and (iv) in $\S 2$.

q. e. d.

LEMMA 3.4. With respect to $\mu(t)$ of (3.4), $\mu(t)<0$ holds for $t \in\left(t_{0}(m), \infty\right)$, where $t_{0}(m)$ satisfies

$$
t_{0}(m)^{m}=\left[\left(m^{2}-4\right)^{1 / 2}-1\right] /\left(m^{2}-5\right)
$$

and $t_{0}(3)<t_{0}(m)<1$. For example, $t_{0}(3)=0.676 \cdots, t_{0}(5)=0.708 \cdots, t_{0}(7)=0.746 \cdots$, etc.

Proof. The solution $t_{0}(m)$ of $\mu(t)=0$ is obtained by calculation. For $1<t$, $\mu(t)<0$ is verified by taking the squares of the both sides of

$$
2 t^{m+1}+t^{1-m}-t<t\left[\left(2 t^{m}-1\right)^{2}+m^{2}-1\right]^{1 / 2} .
$$

LEMMA 3.5. Let $f \in V_{2,0}$ for $\left(S^{m}, g\right)$ and $p u t$

$$
w(t)=2 f \eta+t^{-m} d f \cdot \phi .
$$

Then, $J(t) w(t)=0$ holds on $\left(S^{m}, g(t)\right)$. Furthermore, $w(t)$ is coclosed and $w(t)$ defines a Killing vector field.

Proof. $J(t) w(t)=0$ is verified by (3.2) and relations (i), (iii) and (iv) in $\S 2$. Coclosedness of $w(t)$ is verified by (2.4), (2.5) and $\xi f=0$. To verify that $w(t)$ defines a Killing vector field, it suffices to apply the classical integral formula:

$$
\langle J w, w\rangle+\langle\delta w, \delta w\rangle=(1 / 2)\left\langle L_{X} g, L_{X} g\right\rangle,
$$

where $\langle$,$\rangle denotes the global inner product and X$ denotes the vector field corresponding to $w$.

q. e. d. 
LEMMA 3.6. $\eta$ on $\left(S^{m}, g(t)\right)$ or $\eta_{(\alpha)}$ on $\left(S^{4 r+3}, g(t)\right)$ satısfies the following;

(i) $J(t) \eta=0$,

(ii) $J(t) \eta_{(\alpha)}=4 t\left(t^{m}-2+t^{-m}\right) \eta_{(\alpha)} \quad \alpha=2,3$.

Proof. (i) corresponds to the fact that $\xi$ is a Killing vector field with respect to $g(t)$ for any $t \in(0, \infty)$. To verify (ii), we apply (2.9) and Lemma 3.1 to $J(t) \eta_{(\alpha)}$. q.e.d.

Summarizing the above we obtain the following.

ThEOREM 3.7. $\left(S^{m}, g(t)\right), m=2 n+1 \geqq 3$, is unstable for $t \in\left(t_{0}(m), \infty\right)$, where $t_{0}(m)^{m}=\left[\left(m^{2}-4\right)^{2}-1\right] /\left(m^{2}-5\right)$ and

$$
t_{0}(3)=0.67 \cdots<t_{0}(m)<1 \text {. }
$$

1-forms given in (3.3) are eigen forms corresponding to the negative ergenvalue $\mu(t)$ of $J(t)$.

The contravariant from of (3.3) is obtained by using (2.4); the result is given in the Theorem in the introduction.

If $m=3$, by the deformation $g \rightarrow g(t)$, the eigen forms of $J(0)$ given in Proposition 2.1 are changing as follows;

THEOREM 3.8. On $S^{3}$, as $g \rightarrow g(t)$

(i) $\eta$ remains to be an eigen form corresponding to the eigenvalue 0 of $J(t)$,

(ii) $\eta_{(\alpha)}(\alpha=2,3)$ are eigen forms corresponding to the eigenvalue $4 t\left(t^{3}-2+\right.$ $\left.t^{-3}\right)$ of $J(t)$, which vanishes only at $t=1$,

(iii) $2 f \eta+t^{-3} d f \cdot \phi, f \in V_{2,0}$, is an eigen form corresponding to the ergenvalue 0 of $J(t)$,

(iv) $4 t^{3} f \eta+\left\{3-2 t^{3}+\left[\left(2 t^{3}-1\right)^{2}+8\right]^{1 / 2}\right\} d f \cdot \phi, f \in V_{1}$, is an elgen 1-form corresponding to the eigenvalue $2 t^{4}+t^{-2}-t-t\left[\left(2 t^{3}-1\right)^{2}+8\right]^{1 / 2}$ of $J(t)$.

COROLlaRY 3.9. $\operatorname{Null}(\imath d)=6$ for $\left(S^{3}, g\right), \operatorname{Null}(i d)=4$ for $\left(S^{3}, g(t)\right)$ with $t$ near 1 and $t \neq 1$, and $N u l l(i d) \geqq 8$ for $\left(S^{3}, g\left(t_{0}(3)\right)\right)$.

Remark. To understand the situation of the negative eigenvalue of $J(t)$, it may be helpful to know the range of the sectional curvature $K_{(t)}(X, Y)$ of $\left(S^{m}, g(t)\right)$. The range is given by the following;

$$
\begin{array}{ll}
t^{m+1} \leqq K_{(t)}(X, Y) \leqq t\left(4-3 t^{m}\right) & \text { for } t<1 \\
t\left(4-3 t^{m}\right) \leqq K_{(t)}(X, Y) \leqq t^{m+1} & \text { for } \quad t>1
\end{array}
$$

In fact, with respect to a $D$-homothetic deformation $g \rightarrow g^{*}(\alpha)=\alpha g+\left(\alpha^{2}-\alpha\right)$ $\eta \otimes \eta$, the sectional curvature $K_{(\alpha)}^{*}(X, Y)$ satisfies 


$$
\begin{aligned}
& 1 \leqq K_{(\alpha)}^{*}(X, Y) \leqq H \quad \text { for } \quad \alpha<1 \text {, } \\
& H \leqq K_{(\alpha)}^{*}(X, Y) \leqq 1 \quad \text { for } \quad \alpha>1 \text {, }
\end{aligned}
$$

where $H=(4-3 \alpha) / \alpha$ (cf. Lemma $6.4,(12.1)$ of [11]). We put $\alpha=t^{m}$. By a homothetic change $g^{*}(\alpha) \rightarrow t^{-m-1} g^{*}\left(t^{m}\right)$, we get $g(t)$. Then, the inequalities (3.6) and (3.7) are verified.

For example, if $m=3$, then $\left(S^{3}, g\left(t_{0}(3)\right)\right.$ is $\delta$-pinched, where $\delta=0.1005 \cdots$.

Remark. As for stability or instability of (harmonic mappings of) various Riemannian manifolds, see Howard [1], Howard and Wei [2], Leung [3], [4], Nagano [5], Ohnita [7], Okayasu [8], Pan [9], Urakawa [17], [18], Xin [19], and so on.

\section{REFERENCES}

[1] R. HOWARD, The nonexistence of stable submanifolds, varifolds, and harmonic maps in sufficiently pinched simply connected Riemannian manifolds, Mich. Math. Journ., 32 (1985), 321-334.

[2] R. How ARD AND S.W. Wei, Nonexistence of stable harmonic maps to and from certain homogeneous spaces and submanifolds of Euclidean space, Trans. Amer. Math. Soc., 294 (1986), 319-331.

[3] P.F. Leung, On the stability of harmonic maps, Lect. Notes Math., 949, SpringerVerlag, 1982, 122-129.

[4] P.F. Leung, A note on stable harmonic maps, Journ. London Math. Soc., 29 (1984), 380-384.

[5] T. NAGANo, Stability of harmonic maps between symmetric spaces, Lect. Notes Math,, 949, Springer-Verlag, 1982, 130-137.

[6] М. Овата, Riemannian manifolds admitting a solution of a certain system of differential equations, Proc. U.S.-Japan Sem. Diff. Geom., Kyoto, 1965, 101-114.

[7] Y. OHNitA, Stability of harmonic maps and standard minimal immersions, Tohoku Math. Journ., 38 (1986), 259-267.

[8] T. OKaYAsu, Prinching and nonexistence of stable harmonic maps, preprint.

[9] Y. PAN, Some nonexistence theorem on stable harmonic mappings, Chinese Ann. Math., 3 (1982), 515-518.

[10] R. T. Smith, The second variation formula for harmonic mappings, Proc. Amer. Math. Soc., 47 (1975), 229-236.

[11] S. TanNo, The topology of contact Riemannian manifolds, Illinois Journ. Math., 12 (1968), 700-717.

[12] S. TANNo, Some differential equations on Riemannian manifolds, Journ. Math. Soc. Japan, 30 (1978), 509-531.

[13] S. TANno, The first eigenvalue of the Laplacian on spheres, Tohoku Math. Journ., 31 (1979), 179-185.

[14] S. Tanno, Some metrics on a $(4 r+3)$-sphere and spectra, Tsukuba Journ. Math., 4 (1980), 99-105.

[15] S. Tanno, Geometric expressions of eigen 1-forms of the Laplacian on spheres, Spectra of Riemannian manifolds (Kaigai Pub.), Tokyo, 1983, 115-128.

[16] H. URAKAWA, On the least positive eigenvalue of the Laplacian for compact 
group manifolds, Journ. Math. Soc. Japan, 31 (1979), 209-226.

[17] H. URAKAWA, The first eigenvalue of the Laplacian for a positively curved homogeneous Riemannian manifold, Comp. Math., 59 (1986), 57-71.

[18] H. URAKAwA, Stability of harmonic maps and eigenvalues of the Laplacian, to appear in Trans. Amer. Math. Soc.

[19] Y.L. XIN, Some results on stable harmonic maps, Duke Math. Journ., 47 (1980), 609-613.

Department of Mathematics

Tokyo Institute of TEChNOLOGY 